\title{
Reprendre la leçon, renouer le temps didactique : spiralité du temps du savoir et ajustement contractuel de début de séance en physique seconde
}

To resume the lesson, return to didactic time: Spirality of the didactic time and contractual adjustment at the start of a physics lesson (Fifth Form).

\section{Marie-Pierre Chopin}

\section{OpenEdition}

\section{Journals}

Édition électronique

URL : http://journals.openedition.org/educationdidactique/1619

DOI : 10.4000/educationdidactique.1619

ISSN : 2111-4838

\section{Éditeur}

Presses universitaires de Rennes

\section{Édition imprimée}

Date de publication : 30 novembre 2012

Pagination : $21-40$

ISBN : 978-2-7535-2254-1

ISSN : 1956-3485

\section{Référence électronique}

Marie-Pierre Chopin, «Reprendre la leçon, renouer le temps didactique : spiralité du temps du savoir et ajustement contractuel de début de séance en physique seconde », Éducation et didactique [En ligne], 6-3 | novembre 2012, mis en ligne le 30 novembre 2014, consulté le 08 décembre 2020. URL : http:// journals.openedition.org/educationdidactique/1619; DOI : https://doi.org/10.4000/ educationdidactique.1619 


\title{
REPRENDRE LA LEÇON, RENOUER LE TEMPS DIDACTIQUE : SPIRALITÉ DU TEMPS DU SAVOIR ET AJUSTEMENT CONTRACTUEL DE DÉBUT DE SÉANCE EN PHYSIQUE SECONDE
}

\author{
Marie-Pierre Chopin (LACES-ADS, Université Bordeaux Segalen)
}

\begin{abstract}
Résumé: Larticle convoque le modèle de l'hétérogénéisation (Sarrazy, 2002 ; Chopin 2007, 2011) et les recherches sur la mémoire didactique (Brousseau \& Centeno, 1991) pour mettre en évidence la dimension spiralaire du temps de la diffusion des savoirs dans la classe, rarement prise en compte dans les recherches sur l'enseignement. L'analyse d'une leçon de physique en classe de seconde supporte la démonstration. Elle permet de montrer, par l'examen statistique des mentions temporelles jalonnant la séance observée (évocation du passé, anticipation de l'à-venir), la manière dont professeur et élèves sont co-impliqués dans le projet de construction d'une temporalité commune, nécessitant, en vue de sa clôture, un retour minimal et partagé sur le passé didactique de la classe.
\end{abstract}

Mots clés: Temps de l'enseignement, mémoire, spiralité du temps, contrat didactique, modèle d'hétérogénéisation.

Marie-Pierre Chopin

Comme toute activité sociale, l'enseignement possède une temporalité qui lui est propre: le temps didactique. Ce temps est bien connu des enseignants qui l'expérimentent quotidiennement dans leur classe. Savoir activer tel ou tel ressort pédagogique pour faire avancer la leçon, pressentir le danger d'un aparté trop long auprès d'un élève, ou bien savoir qu'il faudra ici « perdre du temps pour en gagner » selon la célèbre formule de Rousseau ${ }^{1}$... autant d'illustrations d'un ensemble de savoirs mis en œuvre par les professeurs et réglant, souvent tacitement, l'avancée de la leçon. Car le temps didactique est aussi un temps « silencieux $»$. Non réductible au temps physique de l'horloge, il n'est pas non plus comme lui marqué par le cadencement régulier des secondes qui permettrait à l'observateur non averti d'en saisir la manifestation. Non, le temps didactique est un temps moins « visible », difficilement perceptible autrement que par ses effets: l'évolution des connaissances des élèves.

C'est bien à ce caractère silencieux et surtout à la nécessité de son dépassement que nous nous intéresserons dans cette étude. Dans la lignée d'autres travaux déjà menés sur la question ${ }^{2}$, nous proposerons ici de contourner l' « inaudibilité » apparente du temps de l'enseignement pour montrer l'importance d'en saisir d'un peu plus près les logiques fondamentales.

Deux apports principaux seront convoqués: le modèle de l'hétérogénéisation didactique (Sarrazy,
2002, 2008; Chopin, 2007, 2008a) et les travaux sur la mémoire didactique, plus anciens (Centeno, 1995; Brousseau \& Centeno, 1991), toujours poursuivis à ce jour (Matheron 2009; Bouillon 2010). Ce cadrage outillera notre incursion au cœur d'un corpus de données singulier: une leçon de physique en classe de seconde d'un lycée de la région lyonnaise ${ }^{3}$. Cette dernière - c'est ce que nous montrerons - se révélera particulièrement intéressante en effet pour mettre en lumière une caractéristique fondamentale du temps didactique, rarement prise en compte dans les discours sur le temps en éducation: sa « spiralité ». À travers ce concept - dont nous présenterons les particularités dans une première partie - nous proposerons de donner à comprendre sous quelles nécessités et à quel(s) risque(s) les professeurs sont inexorablement conduits à s'engager, à chaque instant de leur enseignement, dans la gestion d'un équilibre subtil et souvent précaire entre passé, présent, et futur didactique. Les implications théoriques et praxéologiques d'un tel phénomène seront mises en discussion.

Enfin, la présentation de cette étude sera marquée par une position particulièrement réflexive relativement au dispositif de recherche engagé pour produire les résultats. Comme l'annonce en effet le cadrage général de cette série d'analyses, la spécificité de ce dispositif (basé sur l'utilisation partagée d'une unité vidéo produite par d'autres chercheurs dans le cadre 
de ViSA) n'aura pas été sans effets sur la manière dont l'étude a pu être menée. Nous en déclinerons quelques-unes des raisons tout au long de ce texte.

\section{Cadrage théorique: hétérogénéisation, mémoire et spiralité du temps}

\section{Temps et hétérogénéisation didactique}

Le modèle de l'hétérogénéisation didactique - on parlera désormais de " modèle d'hétérogénéisation »-concerne les phénomènes de diffusion des savoirs situés à un niveau infra par rapport à celui de l'enchaînement de plusieurs objets du curriculum. Il s'intéresse en effet à ce qui se réalise dans l'espace même d'un chapitre du programme, au sein duquel un ensemble de notions et concepts entrent en résonance pour s'inscrire dans une unité didactique donnée. Par quels moyens, dans l'espace balisé par l'introduction successive de deux objets de savoir, le professeur organise-t-il l'avancée du temps didactique? C'est à cette question que s'attelle le modèle d'hétérogénéisation.

La réponse proposée s'appuie sur une idéeforce: toute avancée de connaissances pour un ensemble d'élèves donné nécessite en premier lieu une hétérogénéisation de leurs positions au sein de l'espace didactique. Le terme d'hétérogénéisation est ici capital. Il souligne que les positions des élèves dans le jeu didactique sont moins considérées comme l'effet de leurs caractéristiques personnelles (sociales ou cognitives), que comme l'effet de leur rencontre avec des situations spécifiques introduites par le professeur. Une telle approche rappelle ainsi la manière dont les anthropologues McDermott et Varenne étudiaient les processus d'institution quotidiens permettant le fonctionnement de nos sociétés. Voilà ce qu'ils écrivaient dans leur célèbre article, «Culture as disability » :

« Les cultures offrent une abondance de positions que les êtres humains doivent occuper. Chaque position exige que son occupant possède, et soit considéré comme possédant, des caractéristiques particulières qui symbolisent, et même légitiment, la réalité de leur position pour les autres. Les gens sont seulement accidentellement nés différents ou très tôt façonnés par la culture pour l'être. Il est plus important de comprendre comment ils sont affectés à ces positions pour être traités différemment ». (Mc Dermott \& Varenne, 1995, traduit de l'américain par. D. Peret et P. Clanché) ${ }^{4}$.

C'est ce processus d'affectation des élèves à des positions didactiques généralement prévisibles qui est au cœur du modèle d'hétérogénéisation. En effet, par l'analyse a priori des situations proposées par le professeur, il est possible d'anticiper les différentes positions didactiques que les élèves seront amenés à occuper tout au long de l'enseignement. Par exemple, lorsqu'un enseignant soumet à sa classe un problème de mathématiques, les caractéristiques de ce dernier (le type de connaissance engagée, la taille des nombres en jeu, etc.) sera déterminante pour prédire les réponses possiblement données par les élèves. Ces dernières, témoignant de rapports au savoir divers $\mathrm{du}$ côté des élèves à l'instant où est soumis le problème, révéleront des degrés variés d'hétérogénéité didactique. Dans les cas les plus triviaux, si tous les élèves répondent de manière similaire, la classe pourra être considérée comme homogène et le problème soumis ne présentera qu'un faible intérêt didactique pour le professeur. Si tous les élèves répondent juste en effet, c'est que les élèves savent déjà. Si au contraire aucun d'entre eux ne peut amorcer une ébauche de réponse, comment évaluer le type de connaissances composant actuellement leur répertoire didactique? Comment dès lors, envisager l'évolution de ces connaissances sous l'influence de situations nouvelles? Pour simpliste qu'il soit, l'exemple illustre assez bien la nécessité à laquelle est rendu tout enseignant: trouver, pour la dynamique même de sa leçon, des points de création d'hétérogénéité didactique. Ainsi, sous le modèle d'hétérogénéisation, les situations proposées au cours d'une leçon auront pour effet d'affecter les élèves à des positions didactiques variées qu'il s'agira de faire évoluer en vue de les rendre conformes à celle définie par l'institution. La mission du professeur sera précisément d'aboutir à cette idonéité des rapports personnels et institutionnels au savoir (Chevallard, 1991). Pour résumer ainsi cette première définition, on dira que le modèle d'hétérogénéisation définit le temps didactique comme un processus de création et de déplacement d'hétérogénéités didactiques s'opérant tout au long d'une séquence d'enseignement. 


\section{Temps et mémoire}

Le modèle d'hétérogénéisation a principalement été développé dans le cadre de séquences d'enseignement définies comme des enchaînements d'un petit nombre de leçons relatives à un même objet de savoir. Dans cette étude, il sera considéré conjointement aux travaux sur la mémoire didactique.

Inaugurés dans les années 80 par Brousseau et Centeno, ces apports enrichissent le modèle d'hétérogénéisation en lui adjoignant la question de la rupture et de la reprise de la leçon au fils de différentes séances composant l'unité didactique. En effet, bien que l'activité d'enseignement épouse officiellement l'axe du temps chronologique, c'est à un travail tout à fait particulier de récupération et d'utilisation du passé que se livre le professeur dans le cours de sa séquence. À ce propos, Centeno allait jusqu'à définir l'enseignement « comme la maîtrise de différents modes de gestion du passé de l'élève dans une situation didactique » (1995, p. 140). Ce travail de manipulation du passé, impliquant une mobilisation des souvenirs et aménageant consubstantiellement les conditions de l'oubli, est ainsi nécessaire à l'avancement de la leçon, c'est-à-dire à la construction du présent et du futur de l'enseignement. Sa mise en perspective avec le modèle d'hétérogénéisation devient de ce point de vue tout à fait nécessaire. Par exemple, si le professeur est conduit à hétérogénéiser et homogénéiser sa classe pour permettre l'avancée du temps didactique, en quoi les ruptures du temps légal au sein d'une séquence d'enseignement impriment-elles une marque particulière sur les modalités de l'avancée du temps didactique dans la classe? Comme le notaient déjà Brousseau et Centeno en effet:

« [L]a durée du temps où certaines formes de savoir peuvent rester en attente est soumise à des lois qu'on ne peut pas ignorer. Par exemple: les leçons ne peuvent pas être coupées par les vacances de manière arbitraire; intercaler d'autres leçons différentes dans un processus où il y a un grand nombre de savoirs non encore institutionnalisés conduit à des ruptures du contrat qui se traduisent en dernier lieu par l'absence d'apprentissage. » (1991, p. 181)

\section{Temps didactique et spiralité}

La réunion des deux corpus théoriques sur la mémoire et l'hétérogénéisation didactique permet ainsi de sonder de manière plus particulière la question de la spiralité du temps didactique.

Clairement nommée sous ce terme dans le modèle d'hétérogénéisation (Chopin, 2007, 2011), plus sous-tendue mais néanmoins fondamentale dans les travaux sur la mémoire, cette spiralité est entendue ici comme une qualité du temps de la diffusion des savoirs. Elle correspond, pour le dire rapidement, à l'imbrication très étroite entre passé, présent et futur de l'action didactique, nécessaire au maintien d'un contrat permettant de satisfaire le procès d'enseignement en cours. Cette image de spiralité entend ainsi rompre d'avec une vision linéaire de l'apprentissage favorisée par la prégnance, dans l'enseignement, de la référence au texte du savoir, et plébiscitée en outre par un grand nombre de discours tournés vers des rationalisations strictement comptables des ressources pour l'enseignement. Non linéaire donc, le temps didactique n'est pas non plus strictement cyclique: ce qu'il faudra en effet viser, in fine, consiste bien en une " avancée » des connaissances des élèves.

En résumé, la spiralité du temps didactique prend acte du fait que l'apprentissage s'effectue par le jeu de la reprise et du réaménagement de l'ancien, en vue de la construction de nouveau. Au cœur de cette logique, c'est bien le flux didactique ${ }^{5}$ de la leçon qui relève d'une économie spécifique, économie assez rarement prise en compte dans les analyses des pratiques d'enseignement. C'est d'elle dont nous traiterons ici, en nous attachant à montrer sa consubstantialité avec l'existence d'une double logique contractuelle pour le professeur: celle le liant à sa classe d'une part (il est mandaté pour faire progresser ses élèves au cours de la leçon); et celle le liant au texte du savoir (qui doit être parcouru pour satisfaire aux objectifs assignés par l'institution).

\section{Données "recueillies"/questions sur les données}

La sélection d'un épisode significatif de la logique spiralaire du temps de l'enseignement a été rendue possible grâce à la mise à disposition d'une unité 
vidéo dans le cadre de la base de données ViSA. Cette vidéo, d'une durée de deux heures, porte sur l'enseignement des spectres d'émission et d'absorption en classe de physique seconde (cf. la présentation de la séance dans le texte d'introduction au numéro). Dans le but de comprendre la manière dont ce corpus a pu conduire à l'étude présentée ici, nous rappellerons rapidement les étapes ayant jalonné son appropriation, depuis le visionnage de l'unité vidéo à la construction d'une contingence nouvelle générée par notre questionnement.

\section{La vidéo d'un autre...}

La position du chercheur débutant le visionnage d'une unité vidéo dont il n'est pas l'auteur est délicate. La seule observation flottante de l'univers qu'il découvre se trouve d'emblée empêchée, ou du moins réduite, du fait de son statut particulier de spectateur visionnant un ensemble de scènes résultant d'un processus de construction technique, théorique et méthodologique auquel il n'a pas accès $a$ priori ${ }^{6}$. C'est donc davantage à un "visionnage flottant » que nous nous sommes trouvée rendue, et par conséquent à un nécessaire processus d'appropriation du matériau.

Dans notre cas, la nature de nos précédents travaux ainsi que les cadres théoriques qui leur sont associés ont «naturellement » guidé la lecture de la séquence d'enseignement proposée. Les premiers visionnages ont ainsi laissé entrevoir ce qu'on peut remarquer d'abord comme un enlisement du temps didactique $^{7}$. Pour le dire succinctement, dans les scènes présentées par l'unité vidéo - notamment au cours de la première heure sur laquelle nous avons concentré notre étude - tout se passait comme si le professeur «n'avançait pas », et ses élèves pas davantage. Le film de la leçon met en effet en scène un professeur visiblement soucieux de clore le chapitre de sa leçon, tentant de mobiliser l'attention et les connaissances de ses élèves dès la reprise de son enseignement. Le comportement de ces derniers pourtant, ne semble pouvoir satisfaire à ses attentes didactiques: agitation et démobilisation gagnent bientôt la classe, qu'il s'agisse d'aspects purement instrumentaux (certains ont égaré leur matériel ou leurs fiches d'activité) ou liés à des enjeux de savoir (les quelques questions posées par les élèves renvoyant à des domaines parfois extérieurs à ceux censés être traités dans cette séance). Quelques exemples significatifs égrènent ainsi la leçon observée:

Épisode 1: Distribution des fiches

[9 mn après le début de la leçon]

Professeur (P): Document 2, il n'en manque à personne?

E1: Monsieur !

P: Julien mets-toi au travail s'il te plaît!

[Bruit des élèves]

P : Qu'est ce qui se passe? Oh oh oh oh oh oh! On discute à deux mais on a le droit de chuchoter !

E2 (s'adresse à P) : [inaudible]

$\mathrm{P}$ : Le document 1 c'était le document avec tous les spectres.

E2: Document 1.

$\mathrm{P}$ : Oui, ben je vais vous le redonner.

E3: Monsieur vous pouvez venir s'il vous plaît?

$\mathrm{P}$ (à un autre élève): Vous l'avez eu ça?

E3: Monsieur vous pouvez venir s'il vous plaît?

[P passe dans les rangs]

E3: [inaudible]

$\mathrm{P}$ : Ben tu vas rattraper avec ton voisin par exemple.

E4: Monsieur, comment ça s'appelle euh... le disque quand il y a plus de lumière et quand...

$\mathrm{P}$ [à un autre élève] : Mais si tu l'as eu le document.

[Puis se retourne vers l'élève qui a posé la question...]

P: Le disque de Newton.

E5 : Monsieur...

P : Qu'est ce qui vous arrive? Vous êtes partis trop tôt mardi ?

E5: Non.

$\mathrm{P}:$ Ah si!

E5: Vous nous avez laissé partir.

E6: En tout cas on est partis sans!

[Les élèves interviennent pêle-mêle].

P: Oui je vais le chercher. Vous l'avez déjà vu ce document c'est le document où il y avait tous les spectres.

E7 : Monsieur c'est quoi?

[P se déplace dans les rangs]

$\mathrm{P}$ : Jeanne non plus t'as pas eu? Toi tu l'as eu?

[P retourne vers son bureau]

$\mathrm{P}$ : Alors prenez le temps de lire... Oh oh oh! On se met au travail! Vraiment!

Épisode 2: Document 1

[13’30 mn] 
P: Chuuuuuuuut! On chuchote s'il vous plaît vraiment! ( $P$ pose son ordinateur sur le bureau) Ca devient difficile de travailler.

E1 : Mais monsieur! Et en fait je ne comprends pas la différence entre les deux dernières questions?

$\mathrm{P}$ : J'arrive... Chuut! Je vous redonne le document 1. C'est un document sur lequel vous avez déjà travaillé.

E (collégialement) : Aaaaaaah! C'était ça! Voilà! Ca me parle plus!

$\mathrm{P}$ : Il y en a un pour deux mais c'est pareil.

E2: Mais je l'ai cherché partout chez moi!

$\mathrm{P}$ : Non mais je pensais que vous vous en souviendriez.

Épisode 3: Collage des spectres [22'15 mn]

P: Chuuuuuuuuuuuttt. Allez vous êtes prêts? Est-ce que vous commencez à avoir tous vos spectres collés sur le modèle?

E (collégialement): Non. Pas tous!

$\mathrm{P}$ : Ça serait bien!

E1: Le rouge qui est à droite?

$\mathrm{P}:$ Le?

E1: Le rouge qui est à droite ???

$\mathrm{P}$ : Alors on reprend ensemble s'il vous plaît.

$\mathrm{P}$ (s'adresse à un élève): Il me semble que tu as des trucs à rattraper et que tu n'as rien compris parce que tu n'étais pas là??

E2: [inaudible]

$\mathrm{P}$ : mais en discutant à trois mètres de là je ne suis pas sûr que ça t'aide beaucoup. Alors moi je veux bien t'aider, faire un petit rattrapage rapide mais c'est maintenant que ça se passe.

\section{Le spectateur et le chercheur}

Sans surprise, la découverte de l'unité vidéo depuis la position de spectateur où nous nous trouvons placés nous conduit à une appréhension des éléments particulièrement saillants dans le déroulement de la leçon. Ici, c'est la difficulté pédagogique à laquelle le professeur est en train de faire face pour conduire ses élèves à entrer dans la leçon qui apparaît « naturellement ». Comment le chercheur doit-il pour sa part interpréter un tel phénomène? Et surtout quel pourrait être l'intérêt d'une telle interprétation?

La question est d'importance dans le cadre de l'analyse d'une vidéo proposée à plusieurs cher- cheurs - elle l'est par voie de conséquence encore davantage dans le cadre de l'usage plus généralisé de la base de donnée ViSA mettant à « disposition » des vidéos de situations d'enseignement. Est-il réellement nécessaire, en effet, de construire une transcription de second ordre de l'épisode proposé, strictement descriptive et donc possiblement redondante eu égard à ce que l'unité vidéo à elle seule pouvait porter? Le chercheur doit-il baser son analyse sur les éléments les plus saillants donnés à voir par l'unité vidéo, devenant ainsi une sorte de commentateur du « match didactique » rediffusé sur son écran? Pour notre part, il s'agissait bien évidemment d'adopter une toute autre posture. La lecture immédiate de l'épisode devait être déconstruite. Encore fallait-il trouver le moyen de faire cela.

Notre démarche fut assez simple. Partant de l'idée selon laquelle tout dysfonctionnement apparent des pratiques (l'enlisement évoqué plus haut par exemple) donne une occasion précieuse d'en comprendre un peu mieux le fonctionnement (Crawford, 2010) ${ }^{8}$, nous avons décidé d'outiller notre approche de ce corpus de manière à pouvoir comprendre la normalité de ce que nous voyions à l'écran. Qu'est-ce à dire? Qu'il était très probable que ce qui se passait dans cette classe en particulier, puisse exister, d'un point de vue fonctionnel (et quoi que de manière moins visible) dans tout processus d'enseignement. Nous n'aurions eu affaire ici qu'à une manifestation particulièrement visible d'une catégorie de phénomènes « vraie » de manière plus générale, mais peu visible à l'œil nu. Ce dernier, plus apte à saisir les dysfonctionnements de surface - ici la démobilisation des élèves ou la désorganisation du cours - que les logiques à l'œuvre, devait être mis à l'épreuve. Il s'agissait, plutôt que d'imputer aux acteurs engagés (visibles sur l'unité vidéo) les causes des troubles survenus au cours de la leçon, de rechercher la pleine fonctionnalité didactique des événements observés.

Toute proportion gardée, une telle approche s'apparente à celle proposée par les anthropologues de l'équipe de Mc Dermott qui travaillait déjà dans les années 70 à partir d'enregistrements vidéo de séances de classe. Voilà ce qu'ils rapportaient à propos de l'un des extraits vidéo soutenant l'une de leurs analyses les plus minutieuses et limité à une scène excessivement courte entre une enseignante et ses élèves: 


\begin{abstract}
"This strip of behaviour has been described as chaotic by many viewers of the film. By pointing to the order in this apparently chaotic behaviour, we will raise the possibility that most behaviour is ordered in ways about which we as observers or participants are systematically inarticulate." (Mc Dermott $\&$ al., 1978, p. 246) ${ }^{9}$
\end{abstract}

\section{L'unité vidéo: dispositif phénoménotechnique}

Comme dit plus haut, nos précédents travaux menés sur la question du temps didactique et la gestion des hétérogénéités dans l'enseignement ont sans aucun doute compté parmi les raisons de notre propre approche de cette vidéo. C'est en effet la question du renouement du temps didactique au moment de la reprise de la leçon qui nous a particulièrement intéressée pour l'analyse. Mais la nature même de l'épisode proposé fut également très déterminante. Si de nombreuses études s'attachent en effet à étudier le temps de l'enseignement à travers la succession de différentes phases (les découpages en synopsis ou en story-board par exemple sont très nombreux dans les études du temps de l'enseignement), la séance proposée ici semblait permettre de mettre en évidence la pleine fonctionnalité didactique des liaisons intra-temporelles - comme les appelle Bensa (2006) - entre les différents éléments structurant le déroulement de l'enseignement:

« Lacte singulier, rappelle l'anthropologue, ne se dissout pas entièrement dans l'éphémère puisqu'il renvoie le plus souvent à un ensemble de faits ayant éclos au cours d'une même période. Il convient donc de mettre en relief les liaisons intratemporelles des actes entre eux. Ainsi se définit un bornage des actions possibles tout au long d'une époque, un régime d'historicité. Au sein de cette aire, les acteurs regardent en arrière et en avant, toujours prêts à réorienter éventuellement leurs actes et leurs propos. Décrire ces mouvements dans le mouvement introduit à un effort théorique spécifique, celui par lequel ces actes, dont le temps est à la fois la matière et le contenant, peuvent être rapportés à une logique commune de devenir. Car tout ne peut arriver à tout moment. Les situations ont leurs règles du jeu. L'horizon d'attente n'est jamais infini. »(Bensa, 2006, p. 142).

C'est donc le régime d'historicité (Hartog, 2003) de la situation d'enseignement observée que nous cher- cherions à mettre en évidence dans notre analyse de l'unité vidéo. Ce qui se passait au cours de cette heure d'enseignement, peu efficace en apparence, devait être compris comme l'effet normal d'une situation débordant largement du cadre du temps légal présenté par la vidéo, c'est-à-dire comme l'effet de la co-présence, dans le cours même de la leçon observée, du présent de l'action didactique, du futur qu'elle cherchait à atteindre, et du passé qui l'avait également façonnée. Avec une telle entrée, notre question de recherche permettait une déconstruction de la lecture immédiate de la vidéo et se confrontait consubstantiellement à une question fondamentale: celle de l'inaccessibilité matérielle des passé et futur liés à cette unité. En effet, comment pouvions-nous mettre en évidence ce qui, a priori, n'était pas « contenu » sur le support proposé, n'y était pas visible au sens strict? Démarcation nette de notre point de vue entre la position du spectateur et celle du chercheur: alors que le premier se trouve empêché de saisir le réel précédant l'épisode visionné, le second peut trouver les moyens, à l'aide d'un cadrage théorique spécifique et d'une méthodologie adaptée, de saisir les marques de cette co-présence de temps non capturés d'un point de vue chronologique, mais présente - et tel fut notre pari dans cette étude - au sein des pratiques données à voir par cette vidéo.

Pour conclure ainsi notre questionnement sur le recueil des «données ${ }^{10}$ ", nous dirons que c'est la pleine dimension phénoménotechnique de ce dispositif aveugle a priori à l'amont et à l'aval de la séance qui a dynamisé l'étude. Le poids de cette absence a obligé le chercheur à rechercher les traces, dans le présent de l'enseignement, de l'action manifeste du passé et du futur. Nous présenterons ainsi dans la suite la manière dont nous avons pu bâtir, à partir d'un corpus partagé, une contingence nouvelle et singulière, propre à notre questionnement.

\section{Méthodologie}

Comme mentionné plus haut, seule la première heure de l'unité vidéo a été utilisée pour l'étude. Elle concentre en effet la plus grande part des dysfonctionnements apparents ${ }^{11}$ de la leçon, et possède en outre une autonomie avérée d'un point de vue didactique - le professeur attend d'ailleurs la fin de cet épisode pour proposer une pause à ses élèves. 
Visionnage É Transcription: vers une étude des interactions verbales

Notre « visionnage flottant » a été suffisamment conséquent pour nous familiariser avec le corpus proposé. Cette familiarisation a rendu de plus en plus manifeste l'importance des interactions verbales entre le professeur et ses élèves, dans un moment où la leçon consiste essentiellement à revenir et à conclure sur des activités réalisées au cours de séances précédentes (cf. introduction du numéro thématique). Cette importance prise par les interactions et surtout leur agencement au cours de l'épisode considéré a ainsi conduit à une retranscription de la totalité de l'épisode, soit une heure d'enseignement ${ }^{12}$.

On pourra noter que de nombreuses difficultés se posent alors d'un point de vue méthodologique puisque le chercheur dépend complètement du dispositif technique mis en place par l'auteur de la vidéo. Dans notre cas, il fut particulièrement difficile de retranscrire les interventions émanant des élèves lorsque ces derniers ne se trouvaient pas à proximité de l'enseignant, seul équipé d'un micro-cravate au moment de l'enregistrement des données ${ }^{13}$.

\section{Recontextualisation}

Une fois la transcription effectuée, une double recontextualisation de l'épisode a été nécessaire: 1/ une recontextualisation temporelle d'une part (l'épisode étudié est extrait d'une séance de deux heures, et fait suite à plusieurs leçons regroupées au sein du même thème au programme de seconde en physique cf. texte d'introduction au numéro) ; 2 / une recontextualisation relative au contenu de savoir d'autre part (une étude épistémologique a été nécessaire pour saisir les enjeux de savoir liés aux interactions verbales entre le professeur et ses élèves ${ }^{14}$ ).

\section{Découpage de l'épisode en 3 phases et 7 sous-phases}

Les deux étapes précédentes ont permis d'identifier plusieurs phases et sous-phases dans l'épisode. Elles rendent compte du type d'activité engageant formellement le professeur et ses élèves dans le cours de la leçon et constituent en outre des marqueurs de l'avancée du temps légal, même si elles ne présentent pas toutes la même durée. Le tableau suivant résume ainsi ces différents moments de la leçon qui constitueront un canevas utile pour les analyses à venir: 


\section{Marie-Pierre Chopin}

\begin{tabular}{|c|c|c|c|}
\hline \multicolumn{2}{|c|}{ Positionnement chronologique } & Nom de la sous-phase & Description sommaire \\
\hline \multirow{3}{*}{$\begin{array}{l}\text { Phase } 1 \text { : } \\
\text { Finir l'activité enga- } \\
\text { gée lors de la séance } \\
\text { précédente } \\
\text { Durée: } 11^{\prime} 45 \\
\left(1^{\prime} 45-13^{\prime} 30^{15}\right)\end{array}$} & $\begin{array}{l}\text { Sous-phase } 1 \\
\left(1^{\prime} 45-4 ' 19\right) \\
2{ }^{\prime} 34\end{array}$ & Lancement de la leçon & $\begin{array}{l}\text { La phase est très largement consacrée à des questions } \\
\text { instrumentales (reprendre son classeur et ses activi- } \\
\text { tés, etc.). Le professeur mobilise ses élèves pour entrer } \\
\text { dans la phase de travail. }\end{array}$ \\
\hline & $\begin{array}{l}\text { Sous-phase } 2 \\
\left(4^{\prime} 19-99^{\prime} 25\right) \\
\text { 5'06 }\end{array}$ & Lancement de l'activité & $\begin{array}{l}\text { L'épisode nécessitera la reprise d'une activité non } \\
\text { terminée par les élèves. Le professeur organise cette } \\
\text { reprise d'un point de vue instrumental toujours, mais } \\
\text { également didactique (il rappelle les enjeux et les } \\
\text { conditions de la réalisation). }\end{array}$ \\
\hline & $\begin{array}{l}\text { Sous-phase } 3 \\
(9 ' 25-13 \text { '30) } \\
\text { 4'05 }\end{array}$ & Travail en groupes & $\begin{array}{l}\text { Les élèves travaillent en petits groupes pour achever } \\
\text { l'activité. Cette phase est particulièrement compli- } \\
\text { quée pour le professeur car les élèves ne semblent } \\
\text { pas être en mesure de faire face à la demande de leur } \\
\text { enseignant. }\end{array}$ \\
\hline \multirow{2}{*}{$\begin{array}{l}\text { Phase } 2 \text { : } \\
\text { Mise en commun } \\
\text { classe entière } \\
\text { Durée: } 14^{\prime} 30 \\
\left(14^{\prime} 30-29^{\prime} 00\right)\end{array}$} & $\begin{array}{l}\text { Sous-phase } 4 \\
\left(14^{\prime} 30-23^{\prime} 10\right) \\
8^{\prime} 40\end{array}$ & Retour au collectif & $\begin{array}{l}\text { Le professeur remobilise l'ensemble de la classe pour } \\
\text { un retour collectif sur un document en particulier } \\
\text { (« le document } 1 \text { »dans le texte de retranscription). } \\
\text { La phase redevient très largement instrumentale. Le } \\
\text { ton changera dans la suivante. }\end{array}$ \\
\hline & $\begin{array}{l}\text { Sous-phase } 5 \\
\left(23^{\prime} 10-29^{\prime} 00\right) \\
5^{\prime} 50\end{array}$ & Reprise professeur & $\begin{array}{l}\text { Dans cette phase le professeur reprend en effet la } \\
\text { main sur l'enjeu didactique de la leçon. Il procède à un } \\
\text { enseignement de type magistral, à partir de questions } \\
\text { posées par les élèves ou de problèmes constatés dans } \\
\text { leur comportement. }\end{array}$ \\
\hline \multirow{2}{*}{$\begin{array}{l}\text { Phase 3: } \\
\text { Bilan et clôture des } \\
\text { activités } \\
\text { Durée: } 26^{\prime} 45 \\
\left(29^{\prime} 00-55^{\prime} 45\right)\end{array}$} & $\begin{array}{l}\text { Sous-phase } 6 \\
\left(29^{\prime} 00-366^{\prime} 45\right) \\
19^{\prime} 00\end{array}$ & Retour sur les activités & $\begin{array}{l}\text { Les élèves sont ensuite invités à faire le bilan des } \\
\text { activités parcourues au cours des différentes leçons. } \\
\text { Le professeur gère très largement ce retour et cette } \\
\text { synthèse. }\end{array}$ \\
\hline & $\begin{array}{l}\text { Sous-phase } 7 \\
\left(36^{\prime} 45-55^{\prime} 45\right) \\
19^{\prime} 00\end{array}$ & Moment de modélisation & $\begin{array}{l}\text { Cette dernière phase est l'occasion pour le professeur } \\
\text { de tenter de faire le lien entre les activités réalisées par } \\
\text { les élèves et les modélisations proposées dans le cours. }\end{array}$ \\
\hline
\end{tabular}

Tableau 1 - Découpage en 7 phases de l'épisode observé

\section{Identifications des mentions au passé, au présent, au futur}

Il s'agissait à présent d'opérationnaliser les informations contenues dans la vidéo dans le cadre de l'étude des conditions du renouement du temps didactique au cours de cette leçon. Le mode de recueil de données que nous avons choisi repose sur l'identification, à partir de la transcription de l'épisode, de l'ensemble des mentions verbales faisant écho au passé, au présent et au futur de la leçon dans le cours de l'enseignement - on parlera désormais de «mention aux temps » dans le déroulement de la leçon. Il s'agit de pouvoir rendre compte de la manière dont le professeur et ses élèves manipulent ensemble leur histoire didactique vécue ou à venir, s'inscrivant en théorie dans un processus commun de construction de connaissances nouvelles.

Des indices de cette action conjointe (Sensevy $\&$ Mercier, 2007) peuvent ainsi être trouvés dans la manière dont professeurs ou élèves se réfèrent 
les uns et les autres à des événements ou éléments survenus, en cours ou encore à venir, dans la leçon, et participant fonctionnellement à son avancée. À titre d'exemple, l'interaction verbale suivante illustre une mention au passée prise en charge par le professeur dans la première partie de sa leçon:

«Il y a 15 jours on disait: "Oh la lumière du jour, la lumière du soleil c'est... ça... Vous vous souvenez? On disait « Bein, c'est continu, il y a un bel arc-en-ciel, c'est de la lumière blanche." .»

Une telle mention au passé, évoquant le résultat d'une observation faite par l'ensemble de la classe au cours d'une activité, enchâsse dans le présent didactique un élément du passé dont on peut penser qu'il permettra au professeur de faire avancer hic et nunc le savoir dans la classe.

Ces mentions aux temps sont ainsi listées dans leur intégralité puis codées. Deux critères sont retenus:

a. Le type de la mention:

- instrumental (I) - le professeur rappelle la fiche dont il faut se saisir pour se préparer à l'activité à venir ou bien le fait que l'on reviendra sur cette question plus tard;

- lié aux activités réalisées (A) - il est fait référence en particulier aux expériences et résultats d'observation lors de séances précédentes;

- lié aux éléments de savoir en jeu dans la leçon $(S)$ - professeur ou élève rappellent une définition, demandent une explication sur une notion importante, etc.

Dans le cas de l'exemple précédemment cité (exemple de "l'arc-en-ciel »), la mention au passé faisait référence aux observations réalisées au cours d'une activité passée. Elle a donc ainsi été codée A. b. Les auteurs de la mention:

- Le professeur prend en charge la mention (P);

- Les élèves sont les auteurs à part entière de la mention (E);

- Professeur et élève(s) participent conjointement à la mention $(C)$.

Pour reprendre à nouveau l'exemple de « l'arcen-ciel ", la mention a été codée P puisqu'elle est portée par le professeur exclusivement.

Notons pour finir que dans les analyses qui suivront, les modalités $E$ et $C$ relatives à l'auteur de la mention seront souvent regroupées pour des raisons à la fois statistiques (faible nombre d'occurrence nuisibles aux calculs) et sémantiques (l'une et l'autre signifient la participation des élèves à la manipulation de l'histoire didactique de la classe).

\section{Résultats et analyses}

149 mentions aux temps ont ainsi été répertoriées au sein d'une matrice. Les résultats suivants sont issus de traitements statistiques réalisés sur ce corpus. Ils visent à mettre à l'épreuve l'hypothèse avancée plus haut à propos de l'importance des liaisons didactiques intra-temporelles au sein de la leçon.

\section{Résultat 1 - L'épisode analysé montre l'existence d'une tension permanente entre passé, présent et futur de l'action didactique}

Le tableau suivant présente la distribution des mentions aux temps (au passé, au présent et au futur) en fonction des 3 phases et 7 sous-phases de l'épisode. Il permet une première lecture dynamique de la leçon: 


\begin{tabular}{|c|c|c|c|c|c|}
\hline \multicolumn{3}{|c|}{ Moment de la leçon } & \multicolumn{3}{|c|}{ Mentions aux temps } \\
\hline Phase & Sous-phase & Passé & Présent & Futur & Total \\
\hline \multirow{13}{*}{1} & & 3 & 2 & 4 & 9 \\
\hline & 1 & $33 \%$ & $22 \%$ & $44 \%$ & \\
\hline & & 7 & 9 & 2 & 18 \\
\hline & 2 & $39 \%$ & $50 \%$ & $11 \%$ & \\
\hline & & 5 & 7 & 3 & 15 \\
\hline & 3 & $33 \%$ & $47 \%$ & $20 \%$ & \\
\hline & & 15 & 18 & 9 & 42 \\
\hline & Total phase 1 & $36 \%$ & $43 \%$ & $21 \%$ & \\
\hline & & 5 & 12 & 1 & 18 \\
\hline & 4 & $28 \%$ & $67 \%$ & $6 \%$ & \\
\hline & & 12 & 8 & 6 & 26 \\
\hline & 5 & $46 \%$ & $31 \%$ & $23 \%$ & \\
\hline & & 17 & 20 & 7 & 44 \\
\hline \multirow[t]{6}{*}{2} & Total phase 2 & $39 \%$ & $45 \%$ & $16 \%$ & \\
\hline & & 14 & 10 & 2 & 26 \\
\hline & 6 & $54 \%$ & $38 \%$ & $8 \%$ & \\
\hline & & 13 & 22 & 2 & 37 \\
\hline & 7 & $35 \%$ & $59 \%$ & $5 \%$ & \\
\hline & & 27 & 32 & 4 & 65 \\
\hline 3 & Total phase 3 & $42 \%$ & $49 \%$ & $6 \%$ & \\
\hline \multicolumn{2}{|c|}{ Total épisode } & 59 & 70 & 20 & 128 \\
\hline
\end{tabular}

Légende :

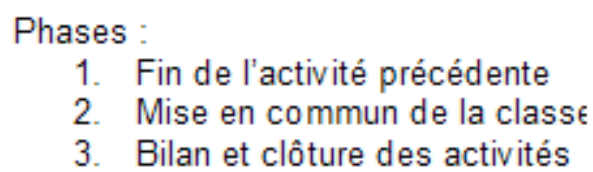

Sous-phases :

1. Lancement de la leçon

2. Lancement de l'activité

3. Travail en groupes

4. Retour au collectif

5. Reprise du professeur

6. Retour sur les activités

7. Moment de modélisation

Tableau 2 - Distribution des mentions aux temps dans la leçon observée

Deux premiers constats peuvent être faits : non seulement toutes les sous-phases de la leçon comportent des mentions relatives au passé, au présent et au futur; mais en outre les lignes « total» des distributions pour chaque phase ( 1 , 2 et 3), font apparaître une certaine stabilité de la répartition des mentions ${ }^{16}$. Dans chaque phase, les mentions au présent sont les plus fréquentes (de 43 à $49 \%$ ), suivies des mentions au passé (de 36 à $42 \%$ ) et enfin des mentions au futur (6 à $21 \%$ ). Notons toutefois que la fréquence de ces dernières diminue logiquement à mesure que l'on approche de la fin de la séance alors que c'est l'inverse qui se passe pour les mentions aux passé: plus la fin de la leçon approche, plus elles augmentent ${ }^{17}$. En résumé, tout se passe comme si la tension entre passé, présent et futur persistait tout au long de l'enseignement, selon un équilibre relativement stable, mais évoluant 
néanmoins vers un épaississement du passé et un évanouissement du futur consubstantiel à l'avancée du temps légal et à la clôture de la leçon.

Si un tel résultat ne surprend pas, il rappelle néanmoins cette nécessité fonctionnelle de la coprésence du passé, présent et futur dans l'action d'enseignement. Nous proposerons maintenant d'aller plus loin pour expliquer les difficultés rencontrées par l'enseignant pour faire avancer le temps didactique dans sa classe.

\section{Résultat 2: Double hiatus entre le temps du professeur et celui des élèves}

Comment en effet comprendre, au vu de ces premiers résultats mettant en évidence les liaisons intra-temporelles de la leçon, que celle-ci avance si difficilement? La réponse que nous apporterons repose sur la mise en évidence d'une sorte de hiatus temporel entre le temps manipulé par le professeur et celui vécu par les élèves.

Pour commencer, l'analyse permet de remarquer que le professeur est le principal protagoniste dans le jeu de mise en écho des temps de la leçon. Sur un total de 149 mentions aux temps, seules 41 sont le fait d'élèves (entièrement ou partiellement ${ }^{18}$ ). Tout se passe ainsi comme si le professeur avançait relativement seul dans la manipulation des temps au sein de la leçon. En outre, lorsque l'on examine de plus près la distribution des mentions au temps réalisées par les élèves, il apparaît que ces dernières se situent très majoritairement dans le présent, alors que le professeur lui, manipulerait en priorité des éléments du passé didactique de la classe. C'est ce dont rend compte le tableau suivant:

\begin{tabular}{ccccc}
\hline & \multicolumn{4}{c}{ Mentions aux temps } \\
\hline \hline & passé & Présent & Futur & Total \\
Professeur & 50 & 43 & 15 & 108 \\
seul & $46 \%$ & $40 \%$ & $14 \%$ & \\
Ėlève & 9 & 27 & 5 & 41 \\
impliqué $^{19}$ & $22 \%$ & $66 \%$ & $12 \%$ & \\
\hline Total & $\mathbf{5 9}$ & $\mathbf{7 0}$ & $\mathbf{2 0}$ & $\mathbf{1 4 9}$ \\
\hline
\end{tabular}

\section{Le hiatus temporel entre professeur et élèves apparaît donc ici de manière significative $\left(X_{2}=12,31 ; p .<.02\right)$. I}

Tableau 3 -Distribution des mentions aux temps en fonction de leur auteur

À ce premier hiatus, s'ajoute un autre décalage significatif entre le travail fourni par les élèves et celui du professeur au cours de l'épisode. C'est ce que montre l'examen du contenu des mentions aux temps. Le tableau suivant rapporte la distribution des mentions portant sur un contenu lié aux activités (A, résultats d'observations par exemple), au savoir ( $\mathrm{S}$, définitions de notions ou de concepts), ou aux aspects plus instrumentaux (I): 


\begin{tabular}{lcccc}
\hline & \multicolumn{4}{c}{ Contenu des mentions aux temps } \\
\hline \hline & Activité & Savoir & Instrumental & Total \\
Professeur seul & 57 & 28 & 23 & 108 \\
& $53 \%$ & $26 \%$ & $21 \%$ & \\
Ėlève impliqué & 13 & 12 & 16 & 41 \\
& $32 \%$ & $29 \%$ & $39 \%$ & \\
\hline TOTAL & $\mathbf{7 0}$ & $\mathbf{4 0}$ & $\mathbf{3 9}$ & $\mathbf{1 4 9}$ \\
\hline
\end{tabular}

Tableau 4 -Distribution des mentions aux temps en fonction de leur auteur et de leur contenu

Comme le montre le tableau 4 , tout se passe comme si professeur et élèves accordaient une place équivalente à l'énonciation du savoir au cours de l'épisode concerné (26\% des mentions y réfèrent pour le professeur, $29 \%$ pour les élèves). En revanche, ils diffèrent fondamentalement quant aux mentions relatives aux activités (surreprésentées chez le professeur, $53 \%$ ) et aux aspects instrumentaux (plus présents chez les élèves, $39 \%$ des mentions ${ }^{20}$ ).

Ce résultat conforte le précédent: l'absence de références aux activités de la part des élèves concorde en effet avec leur tendance à désinvestir le passé de l'action didactique que tente pourtant de faire revivre leur professeur. Cette situation semble paralyser l'avancée du savoir. Ainsi peut-on comprendre l'impression d'enlisement du temps didactique constaté au moment du visionnage de la vidéo. Pour autant, et dans la lignée de notre positionnement théorique présenté en début de texte, nous proposerons maintenant de montrer toute la logique à l'œuvre dans le comportement du professeur et de ses élèves pour sortir coûte que coûte de cet enlisement.

\section{Résultat 3: L'ajustement du contrat permet de renouer le temps didactique}

Bien évidemment, la logique que nous évoquions à l'instant sera appréhendée ici dans sa dimension proprement pratique, ce qui présente l'avantage d'exclure la question de l'intention des protagonistes engagés dans la leçon, et de focaliser l'atten- tion sur les nécessités fonctionnelles du processus d'enseignement.

C'est ainsi sur l'examen de l'apparition chronologique des différents types de mention aux temps que nous clôturerons l'analyse. Celui-ci permet en effet de rendre compte de la dynamique de l'ajustement contractuel qui tente de s'opérer entre le professeur et ses élèves tout au long de l'épisode observé et dont l'aboutissement semble consubstantiel à la sortie heureuse, au sens de la pragmatique d'Austin, de la phase d'enlisement du temps didactique.

Le tableau suivant présente l'ordre chronologique de l'apparition des différentes mentions au passé en fonction de leur type instrumental (I), lié aux activités (A) ou lié au savoir (S) :

\begin{tabular}{lll}
\hline \hline Phase & Sous-phase & Contenu de la mention \\
1 & 1 & Instrumental \\
1 & 3 & Instrumental \\
1 & 3 & Savoir \\
1 & 3 & Instrumental \\
1 & 3 & Instrumental \\
2 & 4 & Activité \\
2 & 4 & Savoir \\
2 & 5 & Savoir \\
3 & 7 & Activité \\
\hline
\end{tabular}

Tableau 5 - Distribution des mentions aux temps en fonction de leur auteur et de leur contenu 
On se souvient que, d'une manière générale, les mentions aux temps réalisées par les élèves sont majoritairement orientées vers le présent (cf. supra Tableau 3). Cet examen de l'apparition chronologique des mentions montre maintenant que, en plus d'être rares, les mentions au passé faites par les élèves apparaissent surtout relativement tard dans le cours de la leçon, la première phase du cours étant majoritairement investie sur le plan instrumental par les élèves. Les deux mentions aux activités en particulier n'apparaissent que dans la sous-phase 4 puis la sous-phase 7 , soit à la toute fin de l'épisode. Tout se passe ainsi comme si il avait fallu «attendre » ces moments tardifs pour acter un renouement des élèves avec le vécu des activités passées. Si celui-ci semble modeste du point de vue de son intensité, on peut remarquer qu'il concorde avec un changement manifeste de contenu dans le type de mention faite par le professeur. En effet, ce n'est qu'à partir de ce moment que les mentions au présent réalisées par le professeur changent de contenu. Jusqu'alors exclusivement instrumentales, elles commencent à porter sur les activités et le savoir en jeu, le professeur entreprenant à ce moment-là l'institutionnalisation de plusieurs éléments liés aux activités.

En résumé, tout se passe comme si le professeur avait « attendu » qu'une part minimale de la classe le rejoigne dans la manipulation du passé pour être libre de réenclencher l'avancée de la leçon dans le présent. Ainsi, s'il n'est pas avéré que les élèves dans leur ensemble aient pu remobiliser leurs expériences issues des activités réalisées, tout se passe néanmoins comme si un retour minimal y avait été nécessaire pour que le présent puisse être réinvesti de nouveaux savoirs par le professeur.

\section{Discussion}

Nous proposerons dans cette dernière partie de revenir sur les résultats principaux de l'étude dans le but d'en discuter quelques implications.

\section{Synthèse et interprétation des résultats dans le cadre du modèle d'hétérogénisation}

Alors que l'épisode proposé laissait apparaître ce que nous avions d'abord reconnu comme un enli- sement du temps didactique (associé à une démobilisation manifeste des élèves), l'analyse a permis de mettre à jour le travail pourtant bien à l'œuvre tout au long de la séance pour permettre une relance effective de la leçon. Tout se passe comme si, sur les aspects à la fois didactiques et pédagogiques (ou sociaux), professeurs et élèves avaient besoin de résorber des hiatus importants pour pouvoir se diriger vers la fin de la leçon.

La nature même de ces hiatus montre, paradoxalement, comment chaque parti poursuit le même objectif que l'autre: clore le chapitre annoncé. C'est sur la nature des moyens utilisés pour le faire que les uns (les élèves) et l'autre (leur professeur), diffèrent. Du côté des élèves, beaucoup paraissent visiblement investis dans les consignes visant à compléter les fiches d'activité distribuées (et conséquemment de coller les spectres); ils posent des questions sur le savoir requis pour pouvoir le faire. De leur point de vue pourrait-on penser, l'énonciation du savoir par le professeur ne vient pas assez tôt, ou pas assez clairement pour leur permettre de comprendre. Une sorte d'impatience se mêle ainsi au chahut qui semble gagner la classe.

Du côté de professeur maintenant les choses en vont tout autrement. Bien sûr, rien ne permet de penser qu'il ne souhaite pas lui aussi clore le chapitre de sa leçon. La pression du temps ne peut qu'être effective pour celui qui tente de satisfaire aux attendus institutionnels relatifs au fait de parcourir le texte du savoir. Néanmoins, l'avancée qu'il appelle de ses vœux ne semble pouvoir être portée par la seule autorité de sa parole. Ce que cherche le professeur consisterait plutôt en une sorte de renouement du temps présent avec le temps passé, passé censé avoir été vécu par les élèves de sa classe. Ce sont les activités qui, pour lui, doivent être remobilisées - comme en témoignent d'ailleurs ses nombreuses références dans une large partie de la leçon. Comment comprendre cet investissement à remobiliser le passé?

D'aucun pourrait lire dans ce phénomène la manifestation d'une caractéristique d'un style pédagogique particulier de l'enseignant (ne souhaitant pas par exemple, imposer le savoir à sa classe, soucieux de rendre ses élèves maîtres des connaissances qu'ils auraient eux-mêmes « construites », etc.). Nous tenterons ici, « profitant » en quelque sorte de l'absence de 
données supplémentaires sur cet aspect ${ }^{21}$, d'avancer une autre hypothèse, plus anthropologique, et largement insufflée par le modèle d'hétérogénéisation.

À l'approche de la clôture de l'enseignement, tout se passe comme si le professeur devait absolument pouvoir prendre acte de l'état des positions didactiques occupées par les élèves relativement aux notions engagées dans le cours. Pour le dire autrement, le contrôle - c'est-à-dire la connaissance et la possibilité de manipulation - de l'état d'hétérogénisation didactique de sa classe apparaîtrait maintenant nécessaire. Pour expliquer ceci, pensons que, avec la fin imminente de l'enseignement, le professeur devra bientôt se livrer à une homogénéisation finale des positions didactiques occupées par les élèves. Ces derniers, en effet, devront bientôt faire un usage des concepts enseignés conforme à ceux que prévoit le programme; leurs positions devront devenir idoines à celle définie par l'institution. Dans une telle perspective, il devient alors plus que jamais nécessaire pour le professeur de permettre à ses élèves de remordre avec le passé (en leur faisant se remémorer les activités par exemple). En effet, ce sont de telles activités qui, au vu de la progression didactique de la leçon, sont censées avoir généré des positions didactiques particulières chez ses élèves; ce sont elles qui ont largement contribué à hétérogénéiser la classe. En cherchant à faire (re)vivre ces hétérogénéités, tout se passe ainsi comme si le professeur se donnait la possibilité d'en prendre la maîtrise, afin de pouvoir envisager la suite et fin de leur déplacement. Dans d'autres travaux auxquels le lecteur sera invité à se reporter pour plus de détails, ce phénomène a été identifié à travers le concept de visibilité didactique (Chopin 2008b) ${ }^{22}$.

\section{Spiralité du temps didactique: un cas limite}

Si cette leçon mise à l'étude semble offrir une bonne occasion de saisir le fonctionnement plus générique de tout processus d'enseignement, c'est qu'elle met en scène de manière particulièrement nette la nécessité de la spiralité du temps de la diffusion des savoirs: sans retour en arrière sur le passé didactique de la leçon, l'avancée présente dans le texte du savoir est compromise.

Une telle idée n'est pas nouvelle. Elle a notamment suscité de nombreux travaux sur la manière dont le professeur manipule la mémoire didactique de sa classe. En particulier, les moments de rappels placés en début de leçon ont fait l'objet d'études minutieuses ( $c f$. par exemple Bouillon 2010), permettant de faire apparaître leurs diverses fonctions (de remobilisation, d'institutionnalisation, etc.). L'intérêt de l'épisode étudié ici tient à ce qu'il présente un cas « limite ${ }^{23}$ de cet usage du rappel comme outil de remobilisation du passé didactique de la classe. En effet, si toute leçon débute par une sorte de « rappel inaugural classique » (« on a vu la semaine dernière que... »), remplissant une fonction à la fois phatique (permettre à la leçon de reprendre) et didactique (permettre de réinvestir le domaine de savoir), la leçon observée dans cet épisode constitue un moment clef de la séquence d'enseignement dans son ensemble, de par la position qu'elle y occupe. Succédant en effet à un ensemble très dense de séances jalonnées d'activités, elle est aussi très proche de la clôture de la leçon où le temps didactique devra être refermé. Arrivés à cette fin de la séquence, on peut comprendre que la jonction avec l'ancien devienne une entreprise particulièrement difficile. Deux raisons au moins seront avancées:

- D'une part, le passé devient lourd de tout ce qui a été vu depuis le commencement de la leçon;

- D'autre part, le futur se charge de plus en plus fortement de la nécessité de "mettre en ordre » ce passé.

C'est cette tension qu'a tenté de gérer le professeur de la leçon observée: en attendant un signe minimal de reprise de l'ancien par les élèves, il pouvait entreprendre à nouveau d'avancer vers la fin de la leçon. Aussi, les difficultés constatables à l'œil nu dans cette entreprise peuvent-elles apparaître comme le signe tangible de la nécessité du maintien d'une logique spiralaire minimale, permettant au professeur de satisfaire au double assujettissement auquel il est contraint dans sa pratique: non seulement il est sommé de parcourir le texte du savoir (celui relatif à la séance étudiée ici a d'ailleurs fait l'objet d'une modification importante due au remaniement récent du curriculum de physique au niveau de la classe de seconde - cf. le texte d'introduction présentant la séance); mais en outre, il (le professeur) se trouve dans l'impossibilité de laisser trop d'élèves sur le côté dans ce cheminement vers la fin de la leçon. 


\section{De quelques implications praxéologiques de cette étude}

Si les résultats obtenus sont finalement peu surprenants au regard des positionnements théoriques adoptés, notons qu'ils génèrent en revanche de nombreuses questions nouvelles à l'issue de ce travail. Nous clôturerons ainsi cette contribution par des remarques sur quelques implications d'ordre praxéologique. Deux domaines seront concernés:

- celui des pratiques d'enseignement (dans le cadre de l'enseignement de la physique en particulier)

- celui des pratiques de recherche à partir d'une base vidéo et du rôle en particulier qu'y jouent les cadres théoriques mobilisés.

L'enseignement de la physique: un temps didactique spécifique?

Pour commencer, ce type de résultat permet de questionner la place et le poids des activités dans l'enseignement de la physique. Si leur fonctionnalité didactique est largement éprouvée dans une appréhension générale des pratiques d'enseignement (la discipline requérant de manière particulièrement manifeste l'observation et le recours à l'expérience), l'est-elle aussi clairement dès lors que l'on adopte une perspective plus dynamique pour approcher le processus d'enseignement? Autrement dit, qu'en est-il de la contrainte exercée par la place, le nombre et l'importance des activités en physique du point de vue de la genèse du temps didactique? Quelle est encore leur ergonomie dans le jeu spiralaire consubstantiel à l'avancée du savoir dans la classe? Comment un professeur peut-il les "récupérer "dans une mémoire didactique commune? Jusqu'à quel point ne menacent-elles pas l'unicité du temps didactique de la leçon, ou, pour le dire avec les mots de Barthes, l'idiorythmie du groupe classe?

Si de telles questions sont - un peu naïvement sans doute - posées en cette fin de contribution, c'est aussi pour souligner que leur réponse, ou du moins leur traitement, permettrait de mettre à l'épreuve des modélisations théoriques à propos du temps didactique généralement issues de l'observation de séances de mathématiques. Dans cette perspective,
ViSA permettrait éventuellement, par un jeu de mise en regard de plusieurs apports théoriques, d'ouvrir un espace nécessaire à leur mise à l'épreuve, voire à leur affinement.

De la même façon, l'enseignement de la physique serait aussi une discipline des plus intéressantes pour examiner les conséquences d'un autre phénomène qui lui est associé: celui de la séparation du groupe classe en deux ou trois sous-ensembles, comme c'est généralement le cas dans les moments de travaux pratiques. Qu'en est-il des effets de cet éclatement du groupe quant à la possibilité donnée au professeur de manipuler le passé des élèves? Quelles questions spécifiques ces situations posent-elles aux modélisations du temps didactique et de sa spiralité?

La recherche à partir de la vidéo d'un autre: le couple modèle/contingence

On le constate, c'est par un ensemble de questions que se termine cette contribution: appel à des croisements théoriques, à des analyses nouvelles, etc. C'est sur un aspect plus épistémologique que nous nous pencherons maintenant.

Comme nous l'avons vu tout au long de l'étude, la dissipation du « désordre » apparent de la situation observée a été largement outillée par des outils conceptuels spécifiques, présents a priori dans le travail du chercheur. Ainsi en a-t-il été pour nous du modèle de l'hétérogénéisation didactique (dans les autres contributions à ce numéro, ce sont d'autres modèles qui ont pu être à l'œuvre). Mais comment (et surtout jusqu'où) assumer, pour le chercheur, un tel " calibrage » de son regardau moment de débuter l'analyse? Comment faire face au risque réel (qui pourrait potentiellement être celui de la base de données ViSA) de ne voir dans toute nouvelle vidéo proposée qu'une « illustration » de plus du modèle préétabli? La question est d'importance, et nous ne prétendrons pas l'épuiser ici en quelques lignes. Pour autant, un retour sur l'étude menée et présentée nous permettra d'esquisser quelques pistes de réponse, que les chercheurs de ViSA auront sans doute à travailler encore longuement ensemble.

En guise d'esquisse donc, nous nous permettrons de souligner l'effort entrepris - peut être trop 
peu traduit au final dans cette forme écrite - pour ne pas donner toute puissance au modèle utilisé pour appréhender la situation d'enseignement. Les protections à la portée du chercheur de ce point de vue ne sont pas nombreuses. Celle utilisée ici aura résidé dans le fait de procéder à un usage que l'on qualifiera d'« heuristique » des cadres théoriques sollicités. En effet, si la théorie aura pu jouer un rôle inspirateur indéniable (c'est elle qui aura, peut-être même à notre insu parfois, orienté notre regard), elle ne nous a pas exempté de la nécessité de la construction d'une contingence nouvelle qui, quoi qu'elle aussi issue d'une opérationnalisation des options théoriques premières, aura permis une mise à l'épreuve effective de ces dernières. Pour le dire plus simplement, en permettant de construire des catégories d'observables (ici les mentions aux temps réalisées par l'enseignant et les élèves tout au long de la séance), les modèles théoriques sollicités (le modèle d'hétérogénéisation et la notion de mémoire didactique) ont généré les moyens de leurs propres mise à l'épreuve: les résultats en effet (tels que la distribution des mentions au temps dans les différentes phases de la leçon), auraient pu invalider les hypothèses de départ et permettant au réel de contrecarrer les prédictions réalisées.

En résumé, plutôt que de « faire parler » le réel par la voix directe de la théorie - toujours propice à «jouer les ventriloques" sur les pratiques des acteurs - nous avons souhaité ici mettre la théorie elle-même à l'épreuve des faits, ceux précisément auxquels l'unité vidéo pouvait permettre d'accéder, ou plutôt ceux qu'elle permettait d'instituer par le truchement d'un regard théorique particulier. C'est essentiellement de cette dialectique féconde entre cadres théoriques et contingence nouvelle que pourra, croyons-nous, émerger la production de résultats robustes et singuliers sur les phénomènes d'enseignement et d'apprentissage dans la perspective dessinée par ViSA. 


\section{NOTES}

1. Voilà par exemple ce qu'écrivait le philosophe dans L'Émile ou de l'éducation: «Oserai-je exposer ici la plus grande, la plus importante, la plus utile des règles de l'éducation? Ce n'est pas de gagner du temps, mais d'en perdre. » (Rousseau, 1966, p. 112).

2. On en trouvera une synthèse critique dans Chopin (2010).

3. Comme nous l'expliquerons plus loin, cette vidéo a été mise à disposition dans le cadre de la base de données ViSA.

4. Texte original: "Cultures offer a wealth of positions for human beings to inhabit. Each position requires that the person inhabiting it must possess, and must be known as possessing, particular qualities that symbolize, and thereby constitute, the reality of their position to others. People are only incidentally born or early enculturated into being different. It is more important to understand how they are put into positions for being treated differently."

5. Nous remercions Abdelkarim Zaid, contributeur à ce numéro, pour la suggestion de cette notion de flux à l'occasion de sa relecture de notre texte.

6. On pourra d'ailleurs souligner que, lors du travail des cinq chercheurs engagés dans cette étude d'une même unité vidéo en vue de la préparation des journées ViSA d'octobre 2011, plusieurs échanges ont été nécessaires, en particulier avec l'auteur de la vidéo, pour apporter des précisions complémentaires aux informations présentes sur la vidéo. Parmi elles, il conviendrait de s'interroger sur celles qui se sont révélées après-coup indispensable aux études de seconde main réalisées, et qui n'auraient pas pu être reconstruites indépendamment de l'accès à l'auteur.

7. Ce « constat » est bien évidemment réalisé a priori, c'est-à-dire qu'il émerge des premiers visionnages de la séance, sans autre médiation que celle du sens commun. À noter qu'il fut en outre partagé par l'ensemble des cinq chercheurs réunis autour du projet d'analyse, au vu d'un certain nombre d'éléments saillants; ils sont déclinés rapidement dans le corps du texte et étayés par des extraits verbatim.

8. Dans son très bel essai intitulé Éloge du carburateur, Matthew B. Crawford, autrefois universitaire, aujourd'hui philosophe et réparateur de motos, raconte à quel point les penseurs et chercheurs de tous ordres manquent souvent de replonger dans la mécanique des phénomènes sur lesquels ils passent leur temps à s'exprimer: « Retour aux fondamentaux, donc » propose ainsi l'auteur. "La caisse du moteur est fêlée, on voit le carburateur. Il est temps de tout démonter et de mettre les mains dans le cambouis... ».

9. [Traduction proposée] «Cette vignette de comportement a été décrite comme chaotique par nombre de visionneurs du film. En faisant émerger de l'ordre dans ce comportement apparemment chaotique, nous mettrons en évidence la possibilité d'ordonner la plupart des comportements qui nous apparaissent - comme aux observateurs ou aux participants - systématiquement inintelligibles. »

10. Dont on se rend compte ici qu'elles sont rarement « données » au sens étroit du mot.

11. Il s'agit bien ici de ce que perçoit le chercheur au visionnage de la séance. Notons que, lors du travail de préparation réalisé par les cinq chercheurs pour cette étude croisée, les mêmes « dysfonctionnements » étaient évoqués dans les discussions. 
12. Notre retranscription a été grandement facilitée par celle déjà mise à disposition par A. Tiberghien sur cet épisode. Ceci souligne l'importance des documents associés aux unités vidéo au moment du référencement dans la base de données.

13. En réalité, il semble que la même séance d'enseignement ait été enregistrée en se focalisant sur les élèves (avec micro positionné de leur côté). Un problème technique survenu aurait rendu inexploitable l'enregistrement. Ce point mériterait plus de développements dans le cadre du fonctionnement de la base de données ViSA autour de quelques questions importantes: celle des unités vidéo « associées » à l'unité visionnées par exemple.

14. On notera qu'une grande part de ces informations ont été réunies, après-coup, c'est-à-dire au cours des différents échanges ayant jalonné le temps de l'étude, par les chercheurs concernés par ce projet d'analyse d'une même unité vidéo. Cela pose donc la question de l'incomplétude possible des informations requises par un chercheur dans le cadre du fonctionnement ordinaire de la base de donnée ViSA).

15. Interruption d'une mn après cette sous-phase (le professeur sort de la pièce pour aller chercher du matériel).

16. Notons que l'augmentation du volume des mentions est largement artificiel puisque les phases ne sont pas d'égale durée (Cf. supra - Tableau 1).

17. Le faible nombre de mentions en phase 3 ne permet toutefois pas de comparer les distributions des phases 1 et 3 (chi-deux non calculable pour cause d'effectif théorique $<5$ ).

18. Les modalités $\mathrm{E}$ et $\mathrm{C}$ ont été regroupées pour les calculs.

19. Élève seul ou élève dans un échange avec l'enseignant.

20. Le calcul du chi-deux renvoie une différence significative à .05 entre les deux distributions.

21. Nous n'avons en effet aucune information supplémentaire sur le professeur. On pourra remarquer que certains chercheurs engagés dans le travail d'analyse sur ce même corpus ont souhaité pour leur part bénéficier de davantage d'informations (cf. la contribution de Laurent Talbot). Il pourrait être intéressant ici de discuter des raisons d’une telle nécessité à « compléter les données».

22. En guise de définition rapide, on dira que la visibilité didactique correspond à la possibilité qu'a le professeur de voir et de contrôler le processus d'hétérogénéisation de la classe. Cette possibilité est ici envisagée dans sa dimension circonstancielle (ou contextuelle) et non en lien avec les capacités personnelles du professeur.

23. C'est-à-dire pour nous, en passe de ne plus être efficient didactiquement parlant (nous en développons les raisons dans la suite du paragraphe). 


\section{RÉFÉRENCES}

Bensa A. (2006). - La fin de l'exotisme. Essais d'anthropologie critique. Toulouse: Anacharsis Editions, 364 p., coll. « Essais».

Bouillon S. (2010). - Temps, culture des professeurs et mémoire didactique. Une étude comparée des modes de gestions de la mémoire dans l'enseignement des mathématiques au collège et à l'école élémentaire. Thèse de doctorat sous la direction du Pr Bernard Sarrazy. Université Bordeaux Segalen, 283 p.

Brousseau, G. \& Centeno, J. (1991). Rôle de la mémoire didactique de l'enseignant. Recherches en Didactique des mathématiques. 11 (2.3), 167-210.

Centeno, J. (1995). - La mémoire didactique de l'enseignant. Thèse posthume inachevée. [textes établis par C. Margolinas. Préface et note de Guy Brousseau]. Université de Bordeaux 1: Ladist. 147 p.

Chopin M.-P. (2007). - Le temps didactique dans l'enseignement des mathématiques. Approche des modes de régulation des hétérogénéités didactiques. Thèse pour le doctorat de l'Université Victor Segalen Bordeaux 2, 337 p.

Chopin M-P. (2008a). - « Approche anthropo-didactique du temps dans l'enseignement des mathématiques: fondements, résultats et perspectives », p. 271-297 In L. Coulange \& C. Hache (Eds), Actes du séminaire national de didactique des mathématiques, Année 2008. Paris: ARDM et IREM de Paris 7, 375 p.

Chopin, M.-P. (2008b). - La visibilité didactique: un milieu pour l'action du professeur. Présentation d'un concept pour l'étude des pratiques d'enseignement. Éducation et didactique, vol. 2, n 2, p. 63-80.

Chopin M-P. (2010). - « Les usages du temps dans les recherches sur l'enseignement ». Note de synthèse. Revue Française de Pédagogie, 170, p. 87-110.

Chopin M-P. (2011) - Le temps de l'enseignement. L'avancée du savoir et la gestion des hétérogénéités dans la classe. Presses Universitaires de Rennes, coll. « Paideia ».

Crawford M. B. (2010). - Éloge du carburateur. Essai sur le sens et la valeur du travail. Paris: Éditions La Découverte, 249 p., coll. « Cahiers libres».

Hartog, F. (2003). - Régimes d'historicité: présentisme et expérience du temps. Paris: Éditions du Seuil, 272 p., coll. « La Librairie du XxI siècle ».

Matheron Y. (2009). - Mémoire et Étude des Mathématiques. Une approche didactique à caractère anthropologique. Rennes: PUR, 219 p., coll. « Paideia ».

Matheron Y. (2009). - Mémoire et Étude des Mathématiques. Une approche didactique à caractère anthropologique. Rennes: PUR, 219 p., coll. « Paideia ».

Mc Dermott R. \& Varenne H. (1995). - "Culture as disability", Anthropology and Education quartely, 26(3): p. 324-348.

Mc Dermott R. P., Gospodinoff K. \& Aron J. (1978). - Criteria for an Ethnographically Adequate Description of Concerted Activities and their Contexts. Semiotica, 24, 3/4, p. 245-275.

Rousseau J.-J. (1966). - Émile ou de l'Éducation. Paris: Garnier-Flammarion, 629 p, coll. « GF ». 
Sarrazy, B. (2002). - Les hétérogénéités dans l'enseignement des mathématiques. Educational Studies in Mathematics, 49, p. 89-117.

Sarrazy, B. (2006). - « Approche anthropo-didactique des phénomènes d'enseignement des mathématiques: fondements épistémologiques et ancrages théoriques », Actes du séminaire national de didactique des mathématiques. Grenoble: La Pensée sauvage éditions.

Sarrazy B. (2008). - « Différencier les hétérogénéités dans l'enseignement des mathématiques: tenants idéologiques et enjeux didactiques » In A. Rouchier et I. Bloch, Actes de la XIII école d'été de Didactique des Mathématiques (Sainte-Livrade, Lot-et-Garonne, du 18 au 26 août 2005). Grenoble: la Pensée Sauvage coll. « Recherches en didactique des mathématiques».

Sensevy, G. \& Mercier, A. (2007). - Agir ensemble. L'action didactique conjointe du professeur et des élèves. Rennes: Presses Universitaires de Rennes, 225 p., coll. « Paideia. Éducation, savoir, société ». 